\title{
SECURE AND EFFECTIVE CONSENT SYSTEM
}

\author{
Hyun-A Park \\ Department of Medical Science, KyungDong University \\ WonJu-City, Kangwon-Do, South Korea
}

\begin{abstract}
The data recorded in a medical information system has a distinctive feature from other kinds of sensitive information. For example in EMR(electronic medical record) of HIS(hospital information system), the data subject is the patient and the persons who generated the medical information about the subject are his doctor and other medical practitioners who perform the treatment prescription of the doctor. Hence, before disclosing the data to outside, doctor's decision and the patient's consent should be made first. However, some researchers have developed secure and cryptographic schemes without considering this feature of medical information. In this paper, we propose a secure and effective consent protocol which is applicable to a real world under the current medical laws, where the server manager cannot guess and disclose the data without the patient's consent and the doctor's decision.
\end{abstract}

\section{KEYWORDS}

Patient's Consent, Doctor's Decision, Data Subject, Data Producer, Data Disclosing

\section{INTRODUCTION}

Generally known as medical information of HIS (hospital information system) or EHR (electronic health records) includes very sensitive and personal things. Nevertheless, it should be devoted as medical data for researches, other statistical surveys or developments of medical systems. The problem at this point is the ownership of data because the data subject is different from data producer in medical information. The data subject is the patient and the producer is his doctor or other medical staffs, so that the methods and processes for managing information and disclosing it to others are different from general sensitive data. When disclosing medical information to the outside, the decision of the data producer and the consent of the patient should be made first, and then the information should be published to the outside after the privacy protection technology is applied.

However, some researchers have proposed security technologies without considering these specificities; the research area of 'patient-controlled (or centered) EHR system' assumes that data owner is the patient and the management/decision on disclosing the data to others is also up to the patient (Szolovits 1994, Eom 2016, Omotosho 2017, Vimalachandran 2017, Seol 2018, Ming 2018, AlThqafi 2016). In (Eom 2016), even a doctor or service provider cannot access the patient's information without patient's consent even if the information was written or produced by the doctor or medical staffs. The authors made it possible by encrypting the information with the patient's private key. That is because the researchers might consider their database model as 'DAS (database as a service), where server manager never know the contents because all cells are encrypted with users' private key, and the data subject and the producer are same as the user. DAS model assumes that server manager is untrustworthy and an inside attacker, so all data are encrypted with users' private key. However, HIS or EHR system have different types of entities; a patient (data subject), a doctor or medical staffs(data producer), a server manager.

In this paper, we propose the consent system for disclosing data to outside, which considers the properties of medical information. Therefore, it can be applicable to current medical laws and systems. 


\section{PRELIMINARIES}

\subsection{Security Definitions}

Definition 1. PRF(Pseudo Random Function) We say that ' $F: K f \times X \rightarrow Y$ is $(t, q, e)$-secure pseudorandom function' if every oracle algorithm $A$ making at most $q$ oracle queries and with running time at most $t$ has advantage $A d v_{A}<e$. The advantage is defined as $A d v_{A}=\left|\operatorname{Pr}\left[A^{F k}=1\right]-\operatorname{Pr}\left[A^{R}=1\right]\right|$ where $R$ represents a random function selected uniformly from he set of all maps from $X$ to $Y$, in which the probabilities are taken over the choice of $k$ and $R$ (Song, 2000).

Definition 2. PRG Gr (Pseudo Random Generator): We say that ' $G_{r}: K_{G r} \rightarrow S$ is a (t,e)-secure pseudorandom generator' if every algorithm $A$ with running time at most $t$ has advantage $A d v_{A}<e$. The advantage is defined as $A d v_{A}=\left|\operatorname{Pr}\left[A\left(G_{r}\left(U_{K G r}\right)\right)=1\right]-\operatorname{Pr}\left[A\left(U_{S}\right)=1\right]\right|$. Where $U_{K G r}, U_{S}$ are random variables distributed uniformly on $K_{G r}$ and $S$ (Song, 2000).

\subsection{Application Scenario}

Our application environment will be applicable to both EHR and HIS, if the shared key is set appropriately. This paper takes one example as an EMR system of HIS. The participants are a doctor, a patient, a server manager, and other medical staffs such as a nurse and an examiner.

Each patient has a private key and the shared keys with other medical staffs. A doctor has his private key and the shared keys with each patient, other medical staffs, and a server manager respectively. A server manager has the institute's secret key and the shared keys with each medical staff. Normally, EMR system is encrypted with the doctors' secret key or the shared key between a doctor and other medical staffs. All private keys and shared keys are registered in advance.

When a request is made for information to be disclosed, the doctor decides whether or not to disclose it and then asks his patients the consent for disclosing data to outside. After decrypting the agreeing data, privacy protection technologies are applied to them.

\subsection{Notations}

Table 1.

\begin{tabular}{|c|l|}
\hline Dr. & doctor \\
\hline Pt. & patient \\
\hline Sr. & server manager \\
\hline $\mathrm{d}_{i} \mathrm{p}_{j}$ & shared key between a doctor $i$ and a patient $j$ \\
\hline $\mathrm{d}_{i} \mathrm{~s}$ & shared key between a doctor $i$ and a server manager \\
\hline$a_{s}$ & $s$-th attribute \\
\hline $\mathrm{r}$ & random number \\
\hline$\alpha_{p j}$ & temporal secret key for patient $j$ \\
\hline$\alpha_{c j}$ & temporal secret key for patient $j$ 's consent \\
\hline $\mathrm{f}$ & pseudo random function \\
\hline S.A & selected attributes \\
\hline A.A & all attributes \\
\hline
\end{tabular}




\section{CONSTRUCTION}

\subsection{Whole Protocol}

1. Notice : disclosing information and requesting consent

2. Dr. $\rightarrow$ Pt. : $\mathrm{y}=\left(\mathrm{x}-\mathrm{d}_{i} \mathrm{p}_{j}\right)+\mathrm{r}_{j}$

3. Pt. $\rightarrow$ Dr. : $f_{\mathrm{r}_{j}}\left(\mathrm{~d}_{i} \mathrm{p}_{j}\left\|S . A\left(a_{1}, \ldots a_{s}, \ldots a_{r}\right)\right\| \alpha_{p j}\right)$

4. Dr. $\rightarrow$ Pt. : $f_{\alpha_{p j}}\left(S . A\left(a_{1}, \ldots a_{s}, \ldots a_{r}\right)\right)$

5. Pt. $\rightarrow$ Dr. : $f_{\alpha_{p j}}\left(\alpha_{c j}\right)$

6. System process : $f_{\alpha_{p j}+\alpha_{c j}}\left(S . A\left(a_{1}, \ldots a_{s}, \ldots a_{r}\right)\right)$

7. Dr. $\rightarrow$ Sr. : $\mathrm{y}=\left(\mathrm{x}-\mathrm{d}_{i} \mathrm{~s}\right)+\alpha_{c j} \| \mathrm{j}$

8. Sr. $\rightarrow$ Dr. \& Pt. : $f_{\alpha_{c j}}\left(A \cdot A\left(a_{1}, \ldots a_{r}, \ldots a_{m}\right)\right)$

9. Dr. \& Pt. $\rightarrow$ Sr. : $\mathrm{y}=\left(\mathrm{x}-\alpha_{c j}\right)+\alpha_{p j}, f_{\alpha_{p j}}\left(S . A\left(a_{1}, \ldots a_{s}, \ldots a_{r}\right)\right)$

10. Sr. : Decrypting S.A and Applying privacy protection technology

\subsection{Protocol Description}

1. The request of consent for disclosing information is noticed to all members of HIS

2. A doctor $i$ challenges each of his patients with an equation; $\mathrm{y}=\left(\mathrm{x}-\mathrm{d}_{i} \mathrm{p}_{j}\right)+\mathrm{r}_{j}$. The patient $j$ inserts the shared key $\mathrm{d}_{\mathrm{i}} \mathrm{p}_{\mathrm{j}}$ to the equation, then he gets the random value $\mathrm{r}_{j}$. This process is a kind of mutual authentication between doctor $i$ and the patient $j$.

3. A patient $j$ sends the encrypted message with $\mathrm{r}_{j}$ to the doctor $i: f_{\mathrm{r}_{j}}\left(\mathrm{~d}_{i} \mathrm{p}_{j}\left\|S . A\left(a_{1}, \ldots a_{s}, \ldots a_{r}\right)\right\| \alpha_{p_{j}}\right)$. This message contains the selected attributes $S . A\left(a_{1}, \ldots a_{s}, \ldots a_{r}\right)$ for the patient $j$ to allow the disclosure, and his temporal secret key $\alpha_{p j}$ which is generated randomly.

4. After receiving the encrypted message, the doctor decrypts it with $r_{j}$ which was generated randomly by the doctor himself in the step 2. He got to know the patient's temporal secret key $\alpha_{p j}$ and encrypted the patient $j$ 's S.A with $\alpha_{p j}$, and sends it to the patient; $f_{\alpha_{p j}}\left(S . A\left(a_{1}, \ldots a_{s}, \ldots a_{r}\right)\right)$. This is also a mutual authentication between the doctor and the patient, and the reconfirming process for the allowed S.A by patient $j$.

5. The patient $j$ verifies the received message and randomly generates another temporal secret key $\alpha_{c j}$ for consent, which is encrypted with $\alpha_{p j}$ and is delivered to the doctor.

6. A doctor $i$ decrypt a patient $j$ 's S.A, then the system automatically encrypts the patient's S.A with a new encryption key $\left(\alpha_{p j}+\alpha_{c j}\right)$

7. A doctor $\underline{i}$ challenge a server manager with $\mathrm{y}=\left(\mathrm{x}-\mathrm{d}_{i} \mathrm{~s}\right)+\alpha_{c j} \| j$. This is also a mutual authentication between doctor $i$ and a server manager. Through this process, the server manager gets the temporal secret key for patient $j$ 's consent. Here, $j$ means the patient $j$ 's ID.

8. The server manager asks doctor $i$ and patient $j$ about the S.A of patient $j$ 's consent through encrypted message $f_{\alpha_{c j}}\left(A . A\left(a_{1}, \ldots a_{r}, \ldots a_{m}\right)\right)$ with $\alpha_{c j}$.

9. The doctor $i$ and the patient $j$ send the equation $\mathrm{y}=\left(\mathrm{x}-\alpha_{c j}\right)+\alpha_{p j}$, which is for mutual authentication through $\alpha_{c j}$ and the provision of another temporal secret key $\alpha_{p j}$ for patient $j$. And they send the encrypted S.A of patient $j$ with $\alpha_{p j}$ respectively.

10. The server manager authenticates mutually with the doctor $i$ and the patient $j$ and finds new value $\alpha_{p j}$. He verifies whether the received messages from the doctor $i$ and the patient $j$ are the same or not. All of the above tests are successful, the server manager decrypts S.A with the secret key $\left(\alpha_{p j}+\alpha_{c j}\right)$ and applies privacy protection technologies to the selected attributes S.A. 


\section{DISCUSSSION AND CONCLUSION}

This protocol is applicable to the current medical laws and medical information systems. At normal time, all data are encrypted with doctor's private key or the shared key between a doctor and other medical staffs, because the doctor or medical staffs are the producer and the manager for the data. However, the data subjects are patients, so that the permission is needed from the data subject when disclosing medical data to the outside. Our proposed protocol shows the doctor's decision and requesting the patient to permit disclosing the data using with their shared key and temporal secret keys. This process enables the server manager not to guess and not to disclose the data without the patient's consent.

As to security respects, the challenging equation makes the mutual authentication and sharing a new key possible. When the shared key in advance is inserted to the equation, the remaining value is a new temporal secret key. The temporal secret keys are generated randomly. With these keys and pseudorandom function, sending messages and data are encrypted. Hence, the security of our protocol depends on it of pseudorandom generator and pseudorandom function.

The proposed protocol is also efficient because all of the used functions are not heavy but light; simple equation and pseudorandom function which falls under the symmetric key encryption method.

Conclusively, our consent protocol can be said available in a real world, secure and efficient. In addition to the consensus system, secure medical information management technologies for normal time and personal information protection technologies for disclosing should be studied to be applicable to current medical laws and medical systems.

\section{ACKNOWLEDGEMENT}

This work was supported by the National Research Foundation of Korea (NRF) grant funded by the Korea Government (Ministry of Education, NRF-2017R1D1A1B03029488).

\section{REFERENCES}

AlThqafi, N. et al, 2016. Achieving Patient-Centered Fine-Grained Access Control in Hospital Information Systems - Using Business Process Management Systems, 9th International Joint Conference on Biomedical Engineering Systems and Technologies, Rome, Italy.

Eom, J. et al, 2016. Patient-Controlled Attribute-Based Encryption for Secure Electronic Health Records System, Journal of Medical Systems, Vol. 40, No.12, pp.1-16

Ming, Y. et al, 2018. Efficient Privacy-Preserving Access Control Scheme in Electronic Health Records System, Sensors, Vol. 18, No.10, doi:10.3390/s18103520

Omotosho, A. et al, 2017. Ensuring patients privacy in a cryptographic-based-electronic health records using bio-cryptography, International Journal of Electronic Healthcare (IJEH), Vol. 9, No. 4, pp. 227 - 254.

Seol, K. et al, 2018. Privacy-Preserving Attribute-Based Access Control Model for XML-Based Electronic Health Record System, IEEE Access, Vol. 6, pp. $9114-9128$.

Song, D. et al, 2000. Practical techniques for searches on encrypted data, IEEE Symposium on Security and Privacy, pp.44-55.

Szolovits, P. et al, 1994. Guardian angel: Patient-centered health information systems, Technical report, Cambridge, MA, USA.

Vimalachandran, P. et al, 2017. Preserving Patient-centred Controls in Electronic Health Record Systems: A Reliance-based Model Implication, ICOT2017, Singapore. 